\title{
Master Function for Analytical Determination of the Interlayer Bond Shear Stiffness and Fatigue Functions for Asphalt Pavements
}

\author{
Borislav Hristov ${ }^{1, *}$ \\ ${ }^{1}$ Department of Engineering Sciences, University of Applied Sciences Berlin, Germany
}

\begin{abstract}
In order to determine the shear stiffness at the interface between asphalt layers and to take into account the interactions of repeated traffic loading, acceleration and braking processes as well as temperature influence, a complicated apparatus for cycling testing of the interlayer bond (CTIB) has been developed. An extensive experimental procedure has been created to include all factors that influence the interlayer bond. Using the experimental results, a master function for the analytical assessment of the shear stiffness has been established. The regression which approximates most accurately the experimentally determined shear stiffness values is the sigmoid function. Through implementation of the master function into a finite element program the fatigue status of asphalt pavements, which is affected by the interlayer bond of different quality, have been calculated over the service life of 30 years using the German method for computational design according to RDO Asphalt 09. The results presented below are based on the results of IGF project "Cyclic Shear Stiffness and Shear Fatigue Testing for Evaluation and Optimization of Interlayer Bond in Asphalt Pavements", supported by the Association of Industrial Research Communities (AIF) of the German Asphalt Institute (DAI) in cooperation with TU Braunschweig.
\end{abstract}

\section{Introduction}

The construction of asphalt concrete pavements usually consists of a surface course, a binder course and a base course. Asphalt base courses are usually produced in two layers when the thicknesses is more than $17 \mathrm{~cm}$. The layers are bonded together through a tack coat, which is usually a bitumen emulsion. The asphalt concrete pavement is loaded permanently in both a vertical direction by vehicle's wheel loads and a horizontal direction by braking and acceleration processes. Additionally, temperature variation also cause additional stresses. The interlayer bond (IB) should be produced as a full-surface and rigid connection between the individual layers of the asphalt pavement in order to allow the transmission of shear stresses between them. The effect of this bond has to be generated through the interlocking of the aggregate particles at the interface, the friction between the surfaces of the two asphalt layers and the adhesion between the asphalt binder of the two

* Corresponding author: Borislav.Hristov@HTW-Berlin.de 
layers and the applied tack coat. When transferring shear stresses across the layer interface, these three factors act simultaneously in different proportions, depending on the asphalt mixes, the temperature, the normal pressure, and the type and quantity of the bitumen emulsion.

Missing or too flexible interlayer bond changes the three-dimensional stress state in the entire pavement structure, which results in a reduced service life due to premature material fatigue. In order to ensure that all layers of the asphalt pavement act as a unit during the load transfer and that no or only very small relative displacements occur at the layer interface between the layers, the production of a best possible rigid interlayer bond must be striven.

A new improved apparatus for CTIB has been designed at the Department of Pavement Engineering at TU Dresden. Its purpose is to simulate as accurately as possible the real load conditions which exist on in-service asphalt pavements. An extensive testing procedure has been created to take into account the interactions of repeated traffic loading, acceleration and braking processes as well as weather-related effects.

\section{Objectives}

The main objective of this study is to develop a master function for analytical assessment of the shear stiffness, which takes into account the dependence between shear stiffness, temperature, shearing frequency and normal stress in order to implement the established master function in the finite element program SAFEM (Semi-Analytical FE Method) [1]. In conjunction with the German method for computational design RDO Asphalt 09 [2] it is aimed to computationally estimate the effect of different shear stiffness functions for qualitatively different interlayer bonds over the service life of the whole asphalt pavement.

\section{Experimental program and development of the master function for analytical assessment of bond shear stiffness}

The experimental program included preparation of double-layered asphalt specimens and their cyclic testing with the newly developed apparatus at different temperatures, normal pressures and shearing frequencies as described in [3].
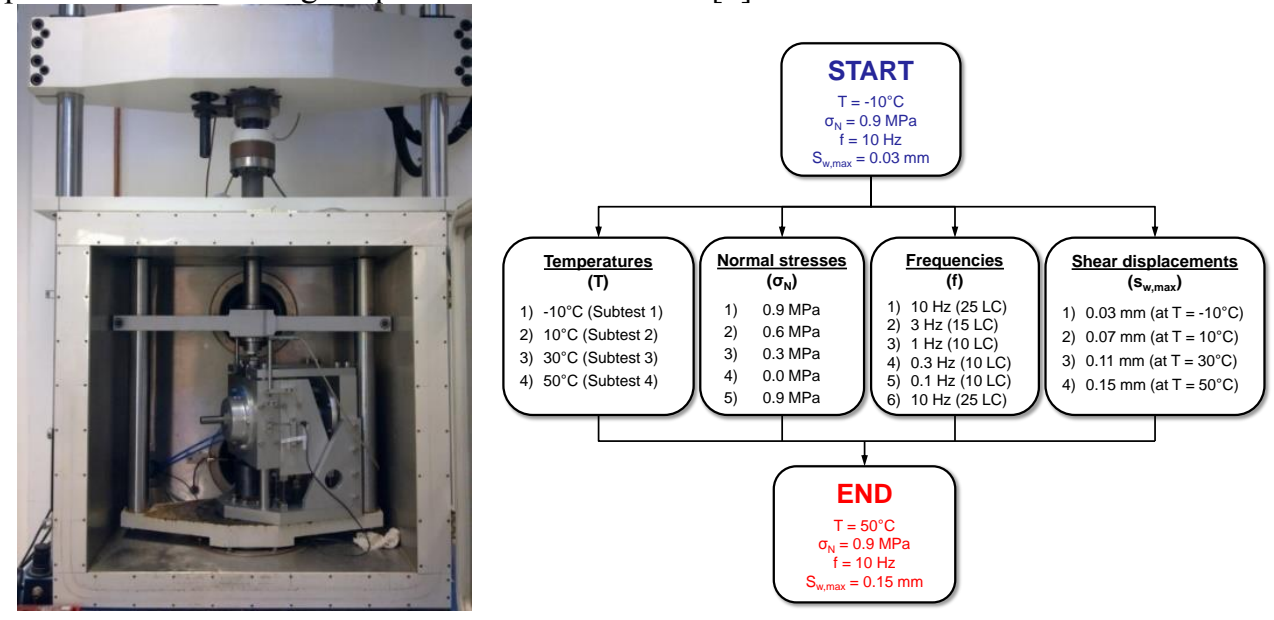

Fig. 1. Testing apparatus for CTIB (left) and testing procedure (right). 
Fig. 1 shows the testing apparatus mounted in the temperature chamber of a servohydraulic testing machine and the fully automated testing procedure for each asphalt specimen.

\subsection{Master function for the shear stiffness}

The shear stiffness at the interface, calculated from the tests is

$$
G_{s}=\frac{\tau_{s}}{\gamma_{s}}=\frac{\tau_{s}}{\left(\frac{s_{w}}{d_{s}}\right)}
$$

with

$$
\tau_{s}=\frac{F_{s}}{A}
$$

where $F_{s}$ is the shear force amplitude $(\mathrm{N}), \gamma_{s}$ is the shear strain $(-), \tau_{s}$ is the shear stress (MPa), $A$ is the cross section at the interface $\left(\mathrm{mm}^{2}\right), s_{w}$ is the shear displacement amplitude $(\mathrm{mm}), d_{s}$ is the gap between steel adapters and thus the interface thickness (mm), and $G_{s}$ is the shear stiffness $(\mathrm{MPa} / \mathrm{mm})$.

In order to develop a universal master function, which takes into account the combined influence of temperature, normal stress and frequency, the relationship between the shear stiffness and the temperature-frequency equivalence was firstly established at the four normal stresses from the average values of at least three tests for every particular test case (Fig. 2).

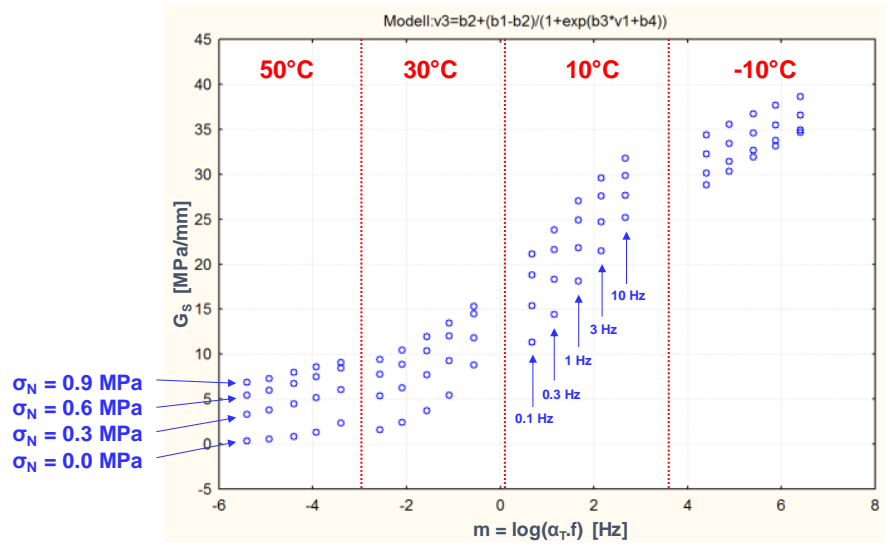

Fig. 2. Functional dependence between shear stiffness and temperature-frequency equivalence at four normal stresses.

The regression used for the master curves which approximated the experimental values most accurately was the sigmoid function. This function approaches asymptotically the minimum and the maximum values of the shear stiffness. The temperature-frequency equivalence was computed as follows:

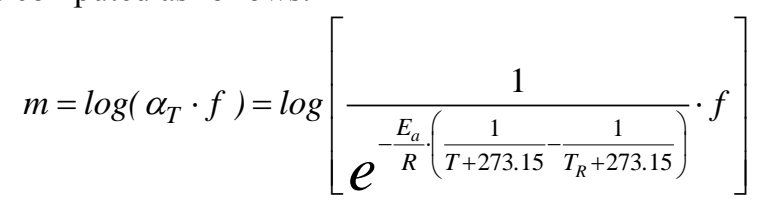


The master function for the shear stiffness $G_{s}$ was found to be

$$
G_{s}=G_{s, \min }+\frac{\left(G_{s, \max }-G_{s, \min }\right)}{1+e^{(m \cdot a+b)}}
$$

where $\mathrm{m}$ is the temperature-frequency equivalence $(\mathrm{Hz}) ; G_{s, \min }$ is the minimal shear stiffness $(\mathrm{MPa} / \mathrm{mm}) ; G_{s, \max }$ is the maximal shear stiffness $(\mathrm{MPa} / \mathrm{mm}) ; \alpha_{T}$ is the shift factor $(-) ; f$ is the frequency $(\mathrm{Hz}) ; T$ is the test temperature $\left({ }^{\circ} \mathrm{C}\right) ; T_{R}$ is the reference temperature $\left(20^{\circ} \mathrm{C}\right) ; E_{a}$ is the activation energy $(\mathrm{J} / \mathrm{mol}) ; R$ is the universal gas constant $(\mathrm{J} / \mathrm{mol} \cdot \mathrm{K})$.

The regression parameters $a$ and $b$ are both functions depending on the normal pressure.

$$
\begin{aligned}
& a=c_{1} \cdot \ln \sigma_{N}+c_{2} \\
& b=d_{1} \cdot \sigma_{N}+d_{2}
\end{aligned}
$$

where $c_{1}, c_{2}, d_{1}, d_{2}$ are function parameters.

After substituting the regression parameters $a$ and $b$ in Equation 4 the equation becomes:

$$
G_{s}=G_{s, \min }+\frac{\left(G_{s, \max }-G_{s, \min }\right)}{1+e^{\left[m \cdot\left(c_{1} \cdot \ln \sigma_{N}+c_{2}\right)+\left(d_{1} \cdot \sigma_{N}+d_{2}\right)\right]}}
$$

Using the master function for $G_{s}(7)$, the shear stiffness of the interlayer bond can be calculated for any arbitrary temperature, frequency and normal stress.

\subsection{Completely fatigued interlayer bond}

From the experimentally determined fatigue curves at the TU Braunschweig it was found, that the increase in the normal stress causes generally an increase in the remaining shear stiffness over the entire service life of the road pavement as well as a higher number of load cycles until complete fatigue (Fig. 3). The fatigue curves approached the same value range at all three temperatures for each normal stress, which leads to the conclusion that the functions of the completely fatigued interlayer bonds are independent of the temperature.

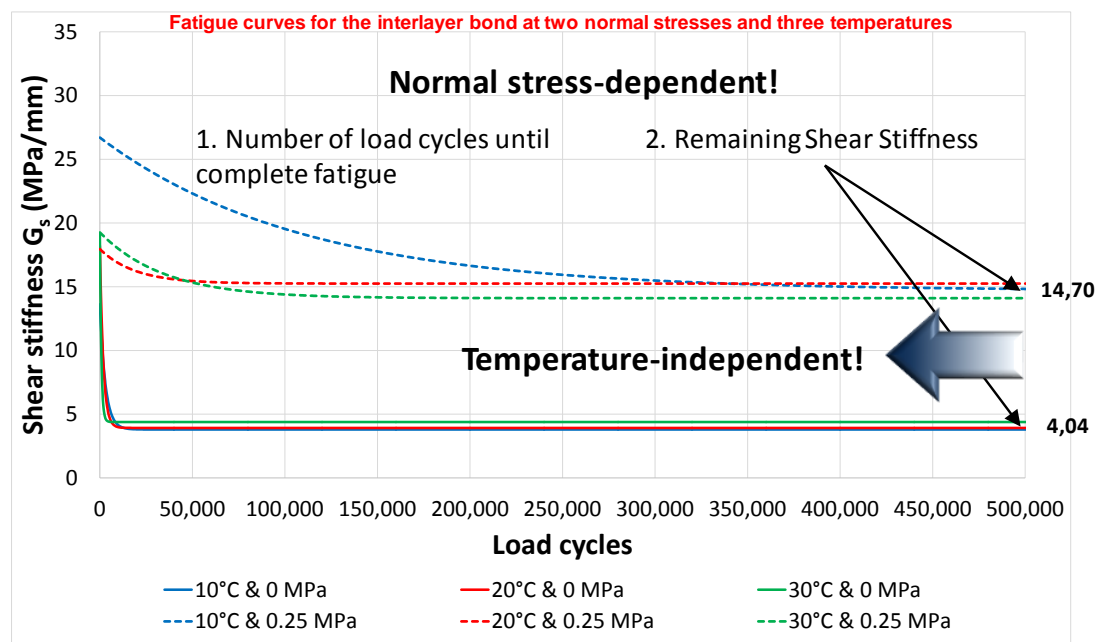

Fig. 3. Fatigue curves for the interlayer bond at two normal stresses and three temperatures. 
Under the assumption that the fatigue is normal stress but not temperature dependent, a linear relationship (8) of the shear stiffness and the normal stress between the average values of the completely fatigued interlayer bonds was determined, namely

$$
G_{S}=42.82 \cdot \sigma_{N}+4.025
$$

Using this function, the service life of the completely fatigued interlayer bond (CFIB) was calculated in SAFEM [1].

\section{Results}

In order to investigate the effects of the reduced (flexible) interlayer bond on the fatigue behavior of asphalt pavements, calculations according to the RDO Asphalt 09 [2] method were performed using the finite element program SAFEM. For this purpose, five different interlayer bond configurations were defined for the layer boundaries between surface and binder course, as well as between binder course and base course:

1. Full interlayer bond (FIB): theoretical case. Upper and lower asphalt layers are firmly connected. It is assumed for the dimensioning calculation according to RDO Asphalt between asphalt layers.

2. Good Case (GC): experimentally established best shear stiffness on laboratoryproduced test specimens,

3. Bad Case (BC): minimum level of shear stiffness on the laboratory-produced test specimens,

4. Completely fatigued interlayer bond (CFIB): determined in long-term tests normalstress-dependent, temperature-independent shear stiffness (please see chapter 3.2)

5. Completely missing interlayer bond (CMIB): theoretical case. Upper and lower asphalt layers slide smoothly on each other.

The cases listed under 1 . and 5. were not proven to exist in the experiments and they are shown purely for orientation. Another question was, what impact a bitumen-based adhesion (flexible and non-rigid interlayer bond) has particularly, taking into account additional interfaces, e.g. for base layers with a thickness of more than $17 \mathrm{~cm}$.

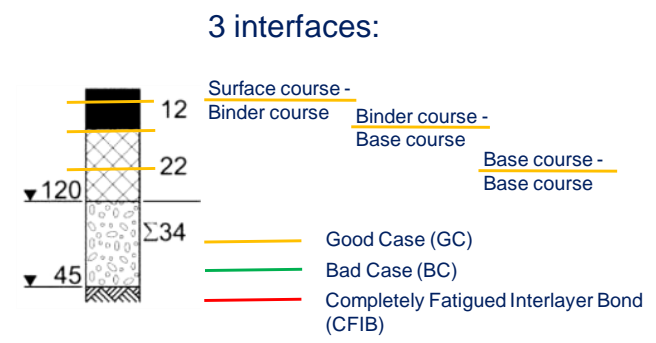

Fig. 4. Possible interfaces

The cases of rigid and completely missing interlayer bond at all interfaces as well as the reference case for 30 years (BC-BC-BC) are always displayed for a better orientation. Figure 5 shows the combination cases BC-BC-BC, BC-BC, GC-GC-GC and GC-GC. A reduction of the service life by $25 \ldots 30 \%$ results solely from the consideration of a third interface. Thus, the consideration of three instead of only two interfaces shows the clear effect of the interlayer bond on the service life of the whole asphalt pavement. 


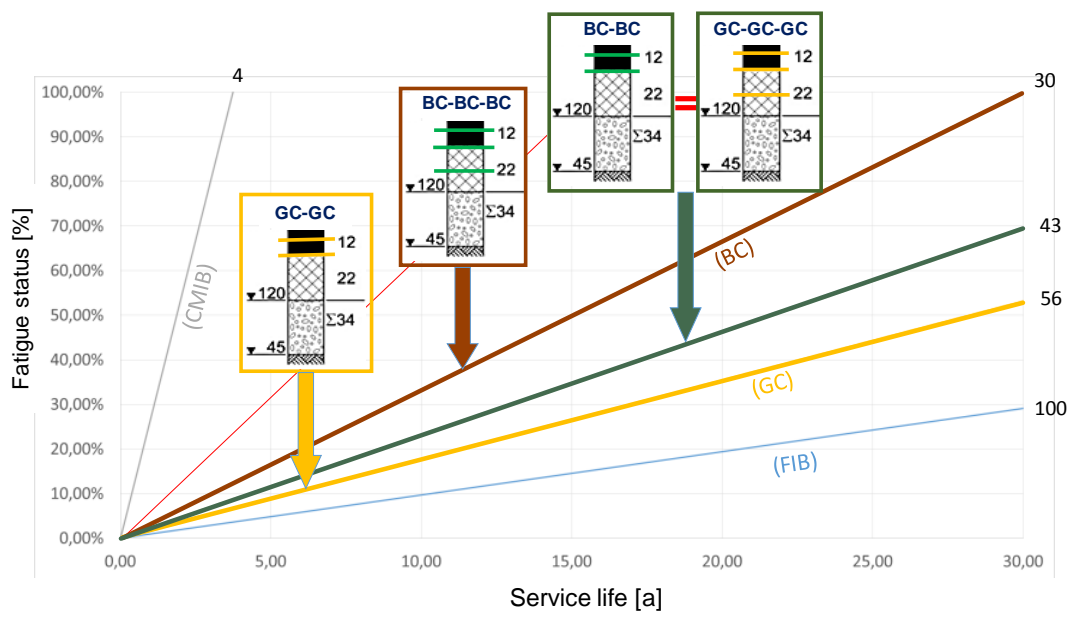

Fig. 5. Comparison between the fatigue functions of three instead of two interfaces under identical conditions.

Almost the same service life has been estimated when using the Bad Case IB with three interfaces as it is in the case of CFIB with two interfaces (surface course on binder course and binder course on base course), as can be seen in Fig. 5. Approximately 50\% service life reduction was calculated for the completely fatigued interlayer bond at all three interfaces, compared to the reference variant BC-BC-BC.

When assuming different variants with a completely fatigued interlayer bond at all three interfaces (Fig. 6), it can be stated, that the interfaces between binder course and base course and between base course and base course cause very similar reductions in the service life.

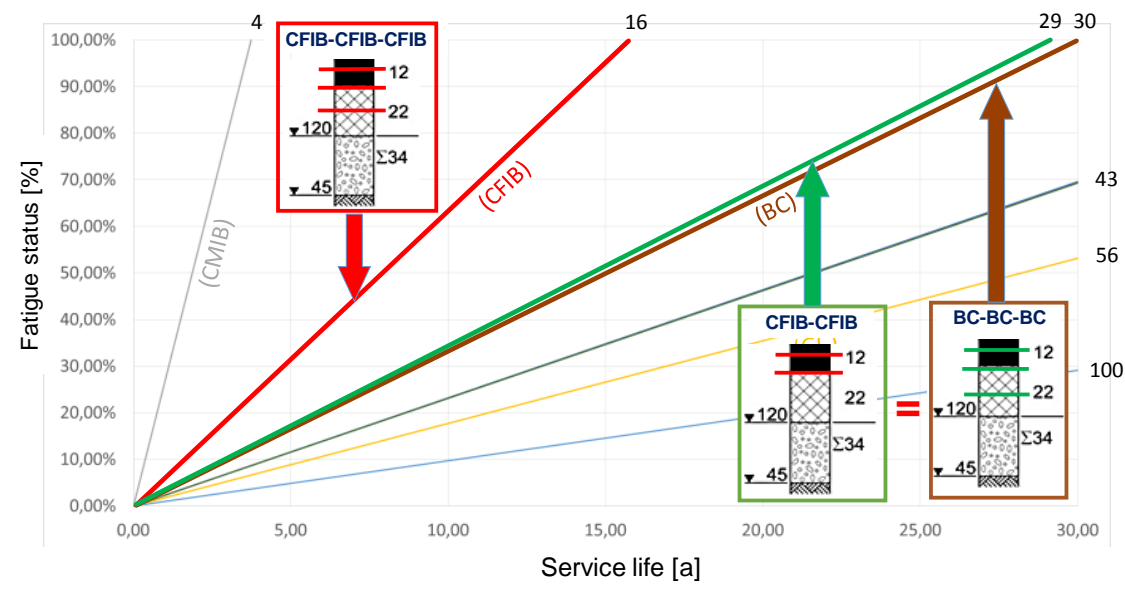

Fig. 6. Interlayer bond combinations between Bad Case and Completely Fatigued Interlayer bond.

Compared to the variant with a completely fatigued interlayer bond at all three interfaces (CFIB), the variant with completely fatigued interlayer bond, calculated in one of the combinations (binder course on base course or base course on base course) hardly shows a change in the estimated service life (Fig. 7). The results show, that the interfaces between binder course and base course and between base course and base course are the relevant ones with respect to the fatigue of the asphalt base courses. 


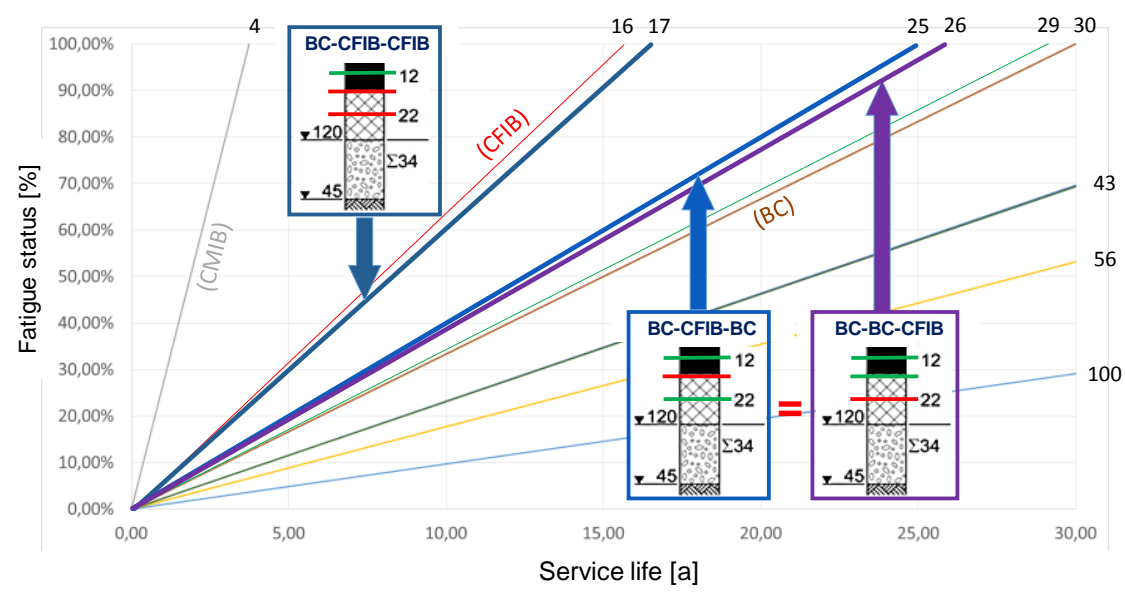

Fig. 7. Other interlayer bond combinations between Bad Case and Completely Fatigued Interlayer bond.

\section{Conclusions}

A master function for the analytical assessment of the interlayer bond shear stiffness has been established on the basis of results from performance testing of the interlayer bond. This master function has been implemented in the semi-analytical finite element program SAFEM [1] to estimate the influence of the interlayer bond on the service life of asphalt pavements and takes into account the combined influence of temperature, normal pressure and shearing frequency. Using the finite element calculations and applying the German method for computational design according to RDO Asphalt 09 [2] fatigue functions for a full (FIB), a good (Good Case), a most achievable (Bad Case), a completely fatigued (CFIB) and a completely missing (CMIB) interlayer bond have been established for an exemplary assumed asphalt pavement. The detailed calculations presented in this paper show the change in the service life of asphalt pavements caused by the interlayer bond of different quality and combinations.

The author would like to thank the German Federation of Industrial Research Associations "Otto von Guericke" (AiF) for financial support.

\section{References}

1. M. Oeser et al., Verbesserung der Sicherheit und Wirtschaftlichkeit der rechnerischen Dimensionierung von Asphaltbefestigungen über ein Finite Elemente Modell. FE 04.0259/2012/NGB, BASt (2014)

2. Forschungsgesellschaft für Straßen- und Verkehrswesen (FGSV), Richtlinien für die rechnerische Dimensionierung des Oberbaus von Verkehrsflächen mit Asphaltdeckschicht RDO Asphalt 09, Köln (2009)

3. B. Hristov, Influence of Different Interface Properties on the Interlayer Bond Shear Stiffness. IOP Conf. Series: Materials Science and Engineering, Volume 365, (2018)

4. F. Canestrari, E. Santagata, Temperature effects on the shear behaviour of tack coat emulsions used in flexible pavements. The International Journal of Pavement Engineering, Vol. 6, No. 1, pp 39-46 (2005) 
5. B. Hristov, F. Wellner, Assessment of the interlayer bond behavior through cyclic shear tests and development of fatigue functions to forecast the service life of asphalt pavements. 10th ICPT 2017, Hong Kong (2017)

6. B. Hristov, F. Wellner, Numerically Supported Experimental Determination of the Behavior of the Interlayer Bond in Asphalt Pavements. Transportation Research Record: Journal of the Transportation Research Board, Vol. 2, Issue2506, Transportation Research Board of the National Academies, Washington, D.C., pp 116125 (2015)

7. B. Hristov, F. Wellner, The Effect of Interlayer Bond on the Service Life of Asphalt Pavements. Global Science and Technology Forum (GSTF), Singapore, pp 444-449 (2016)

8. L. N. Mohammad, Z. Wu, A. Raqib, Investigation of the Behavior of Asphalt Tack Coat Interface Layer. Louisiana Transportation Research Center (2005)

9. G. A. Sholar, G. C. Page, J. A. Musselman, P. B. Upshaw, H. L. Moseley, Preliminary Investigation of a Test Method to Evaluate Bond Strength of Bituminous Tack Coats. Journal of the Association of Asphalt Paving Technologists, Vol. 73, pp 771-806 (2004)

10. F. Wellner, B. Hristov, U. Reinhardt, Beeinflussung der Nutzungsdauer durch den Schichtenverbund. In Asphalt Vol. 01/2017, pp 12-19 (2017)

11. M. A. Miner, Cumulative damage in fatigue, Journal of Applied Mechanics. Vol. 12 (1945) 